\title{
Leishmaniasis in the era of tumor necrosis factor alpha antagonist therapy - a research agenda for Europe
}

P Zanger (philipp.zanger@uni-tuebingen.de)1 ${ }^{1}$ S Gabrysch ${ }^{2}$

1. Institut für Tropenmedizin, Eberhard Karls Universität, Tübingen, Germany

2. Institut für Public Health, Ruprecht Karls Universität, Heidelberg, Germany

Zanger P, Gabrysch S. Leishmaniasis in the era of tumor necrosis factor alpha antagonist therapy - a research agenda for Europe. Euro Surveill.

2013;18(30): pii=20542. Available online: http://www.eurosurveillance.org/ViewArticle.aspx?Articleld =20542

A number of published case reports suggest an association of tumor necrosis factor (TNF) alpha antagonist use and manifest leishmaniasis. Despite increasing popularity of antagonising TNF alpha for the treatment of autoimmune disorders, systematic research on the risk of opportunistic leishmaniasis in patients receiving these drugs is lacking. This perspective identifies areas of uncertainty regarding the safety profile of TNF alpha antagonist drugs and their clinical use in patients at risk of leishmaniasis. Then, we reflect on how current pharmacovigilance activities in Europe could be enhanced to help reduce these uncertainties. Our aim is to stimulate a debate about this important drug safety issue with potential consequences for patients receiving TNF alpha antagonists living in or travelling to areas endemic for leishmaniasis.

\section{Introduction}

Since their introduction about a decade ago, tumour necrosis factor (TNF) alpha antagonist drugs have greatly improved the clinical management of autoimmune disorders and are now widely used in rheumatology. The flipside of the coin is an increase in infectious disease risk in patients treated with these drugs. Both reactivation of latent infections and increased susceptibility to new infections have been observed in patients receiving TNF alpha antagonist therapy. Depending on their mode of action, the TNF alpha antagonist drugs most widely used can be distinguished in two major groups: monoclonal antibodies, such as infliximab and adalimumab, and the receptor construct etanercept [1].

Leishmaniasis is endemic in large parts of southern Europe [2-5] where studies suggest a focal prevalence of latent infection of up to $53 \%$ in the adult population [5]. Two species, Leishmania infantum and L. tropica are transmitted around the Mediterranean basin by the bite of sandfly species (order Diptera, family Psychodidae, subfamily Phlebotominae) [4]. The spectrum of manifest infection ranges from a well localised, self-healing cutaneous papule or ulceration to rapidly fatal visceral disease. Host immunity is known to be a key determinant of the clinical manifestation and outcome. Immuno-suppression, in particular conditions that alter the type 1 helper T-cell-mediated immune response of which TNF alpha is an important component, is generally regarded as a risk factor for manifest leishmaniasis and has been identified as a major contributor to its re-emergence in Europe [4]. A number of published case reports suggest an association of TNF alpha antagonist use and manifest leishmaniasis $[6,7]$. Despite this, no systematic research on opportunistic leishmaniasis in patients receiving TNF alpha antagonists has been conducted.

Based on a brief summary of published information on opportunistic leishmaniasis and use of TNF alpha antagonists, this perspective identifies areas of uncertainty regarding their safety profile and their clinical use in patients at risk of leishmaniasis. We then reflect on how current pharmacovigilance activities in Europe could be enhanced to help reduce these uncertainties. Our aim is to stimulate a debate about this important drug safety issue with potential consequences for patients receiving TNF alpha antagonists living in or travelling to areas endemic for leishmaniasis.

\section{Current knowledge on tumor necrosis factor alpha antagonist therapy and leishmaniasis}

Published research on therapy with TNF alpha antagonists and leishmaniasis is scarce and limited to case reports and case series. We recently reviewed the literature [6] and identified 19 descriptions of patients with leishmaniasis while receiving TNF antagonists, published between 2004 and 2011. Nearly all of the identified cases (18/19) occurred in Europe, and more than two thirds were published in 2010-11. The vast majority were reported from endemic regions of southern Europe (14/18) whereas fewer occurred after travel to and migration from endemic areas (4/18). The reported time period from initiation of treatment to onset of leishmaniasis ranged from 0.5 to 48 months, consistent with both reactivation of latent infection and increased susceptibility to new infection.

Only one reported case was treated with etanercept while all others received either infliximab or 
Aims and objectives of a coordinated research effort on opportunistic leishmaniasis in patients treated with tumor necrosis factor alpha antagonists in Europe

\section{Aims}

- Estimate the impact of TNF alpha use on the occurrence of leishmaniasis in Europe.

- $\quad$ Reduce leishmaniasis risk by tailoring TNF alpha antagonist therapy to individual risk profile.

- Optimise anti-parasitic and anti-rheumatic therapy in patients with opportunistic leishmaniasis.

\section{Objectives}

- Define the absolute risk of leishmaniasis in patients treated with TNF alpha antagonists. Define the relative risk of leishmaniasis in patients treated with TNF alpha antagonists

- $\quad$ with respect to classic immunosuppressive regimens; and

- with respect to type of TNF alpha antagonist used.

- Define the proportionate contribution of new infection and reactivation towards the burden of leishmaniasis in patients treated with TNF alpha antagonists

- overall; and

- by type of TNF alpha antagonist used.

- Define the role of screening (serology, intradermal leishmanin) in patients treated with TNF alpha antagonists for clinical decision making by determining their predictive values

- with regard to the overall risk of leishmaniasis; and

- depending on the type of TNF alpha antagonist used.

- Identify groups at risk of and factors associated with developing leishmaniasis during TNF alpha antagonist therapy namely

- environmental/behavioural (e.g. companion animals, travel, region of residence) factors;

- host factors (e.g. co-morbidity); and

- others (e.g. immunosuppressive co-medication)

- Optimise therapy of leishmaniasis as complication of TNF alpha therapy through either

- $\quad$ interruption or continuation of TNF alpha antagonist; and

- $\quad$ systemic or local treatment of cutaneous leishmaniasis.

- Explore whether TNF alpha antagonists can be restarted after cure of leishmaniasis and if

- $\quad$ there is a need for potential modifications of this therapy;

- treatment can be continued with identical or alternative TNF alpha antagonist;

- $\quad$ immunosuppressive co-medication needs to be modified; and

- anti-parasitic maintenance therapy is necessary to prevent relapses (secondary prevention).

TNF: tumor necrosis factor.

adalimumab. This was surprising, since prescription data from the countries where the cases occurred showed that each of the three drugs was prescribed about equally often. These findings suggest that opportunistic leishmaniasis is more likely to occur in patients receiving TNF alpha monoclonal antibodies than in patients treated with etanercept. This interpretation is supported by similar findings for tuberculosis $[8,9]$ and by studies in mice [10] and in vitro [11].

Interestingly, published case reports describe various approaches including discontinuation as well as continuation of TNF alpha antagonist therapy during and after anti-parasitic treatment while using the same or a different type of drug for ongoing TNF alpha antagonisation [6]. Their number, however, is too small and the data and observation period reported too heterogeneous to decide whether one or the other approach is associated with an increased risk of recurrent leishmaniasis.

Although it provided us with a hypothesis on the possible differences of leishmaniasis risk by type of TNF alpha antagonist used, our analysis of case reports is limited by potential publication bias and confounding underlying the observed associations. Moreover, the number of published cases is likely to represent only a small fraction of patients treated with TNF alpha antagonists with opportunistic leishmaniasis, leaving the magnitude of this drug safety issue unclear. Hence more systematic research is needed to improve our 
understanding of opportunistic leishmaniasis in these patients.

\section{Unsolved questions}

The main areas of uncertainty pertaining to opportunistic leishmaniasis in patients receiving TNF alpha antagonist therapy, to be addressed by future research, are outlined below and summarised in Box 1 .

There is a clear need to estimate the potential impact of TNF alpha antagonist use on the incidence of leishmaniasis. This information is required to clarify to what extent there is a need to further investigate and corroborate this association and to justify funding of research activities that aim at elucidating risk factors for leishmaniasis among those treated with TNF alpha antagonists.

Any study on risk factors should attempt to answer whether there truly is a difference in risk of leishmaniasis depending on the type of TNF alpha antagonist used. Once supported by evidence from prospective studies, physicians (mainly clinical rheumatologists) could directly translate this knowledge into practice by choosing the TNF alpha antagonist with the lowest risk for opportunistic leishmaniasis for patients living in or travelling to endemic areas. Moreover, the role of behavioural and host-associated risk factors for clinically manifest leishmaniasis under TNF alpha antagonist therapy should be evaluated as knowledge gained could be relevant in clinical decision-making or counselling of patients.

Once a risk difference for the various types of TNF alpha antagonists has been established, it will be important to clarify if this is due to reactivation of latent leishmaniasis or increased susceptibility to new infection.

It needs to be determined whether screening for latent infection before initiation of TNF alpha antagonist therapy can play a role in preventing opportunistic leishmaniasis. Studies evaluating the predictive values of serologic and intradermal leishmanin testing have great potential to inform clinical decision-making and will equally contribute to our understanding of latent versus newly acquired infection for the onset of opportunistic leishmaniasis in patients receiving anti-TNF alpha therapy.

Apart from defining risk factors, there is a need for clinical research to improve medical care for patients suffering from opportunistic leishmaniasis. In particular, better evidence on whether TNF alpha antagonists can be continued in patients with clinically active leishmaniasis, or after its cure, is of high clinical relevance since many of these patients depend on TNF alpha antagonists to adequately control the underlying autoimmune disease. Besides, future research has to address whether there is a need for secondary prevention of opportunistic leishmaniasis in patients that require sustained antagonisation of TNF alpha.
For instance, type and duration as well as indicators for the initiation of anti-parasitic maintenance therapy need to be established. Of note, anti-TNF alpha therapy has been continued or re-initiated in several cases of opportunistic leishmaniasis without subsequent relapse $[6,12]$, indicating that a first episode does not justify long-term anti-parasitic treatment.

Finally, we have to learn more about the risk of generalised infection secondary to localised cutaneous leishmaniasis in patients receiving TNF alpha antagonists. Published case reports indicate that most clinicians fear this complication and opt for systemic anti-parasitic therapy. This, however, has to be balanced against increased toxicity when compared to local treatment which could be an option in a setting where close monitoring of the patient is ensured.

Research challenges concerning opportunistic leishmaniasis and tumor necrosis factor alpha antagonist therapy

Traditionally, spontaneous notification of adverse events to national pharmacovigilance systems has been used to define a drug's safety profile with regard to rare events and long-term effects that may have remained undetected during pre-licensure clinical trials. This approach, however, is subject to significant underreporting, does not allow analysing the number of reported events relative to the number of subjects treated, and cannot provide an estimate of the baseline risk.

In response to these shortcomings and with the support of the pharmaceutical industry, national rheumatology societies in several European countries have initiated national drug registers as post-marketing surveillance tools [13]. Many of these were put in place simultaneously to the licensing of the first TNF alpha antagonists about a decade ago. Their methodologies were recently reviewed in detail by Zink et al. [13]. In brief, selected care providers in rheumatology enrol patients receiving TNF alpha antagonists or other biotherapies into epidemiological cohort studies or into registers, thus overcoming some of the limitations of traditional pharmacovigilance activities.

Although a huge improvement compared to the traditional reporting systems, it has been called into question whether national drug registers cover a sufficient patient base to detect rare adverse events. Zink et al. estimate that events with an incidence equal or below one in 1,000 patient-years may not be adequately detected by this approach [13]. With regard to leishmaniasis, we observed that the French register only detected half of leishmaniasis cases reported in publications from France over a defined time period [6], implying that underreporting may not be adequately addressed by the national registers. Besides, none of these registers had detected sufficient numbers of cases to allow analysing whether leishmaniasis risk 


\begin{tabular}{|c|c|}
\hline Component & Function/role \\
\hline $\begin{array}{l}\text { Coordinating board hosted by } \\
\text { European institution including representatives } \\
\text { from existing European network structures } \\
\text { in clinical parasitology/ tropical medicine, } \\
\text { dermatology, rheumatology, infectious diseases } \\
\text { and supported by public health experts }\end{array}$ & $\begin{array}{l}\text { - Design of post-authorisation safety studies (cohort studies) and observational clinical } \\
\text { studies } \\
\text { - Ensure uniform methodology and data collection } \\
\text { - Enhance national drug registers in countries endemic for leishmaniasis (where } \\
\text { - Hocessary) } \\
\text { migrants) } \\
\text { - Increase awareness among policy-makers and professional societies }\end{array}$ \\
\hline $\begin{array}{l}\text { National drug registers hosted by professional } \\
\text { societies in rheumatology }\end{array}$ & $\begin{array}{l}\text { - Implementation of cohort studies } \\
\text { - Increase awareness/ case reporting among clinicians }\end{array}$ \\
\hline $\begin{array}{l}\text { Institutions specialised in diagnosis and } \\
\text { therapy of leishmaniasis, represented through } \\
\text { existing European network structures in clinical } \\
\text { parasitology/ tropical medicine, dermatology, } \\
\text { infectious diseases }\end{array}$ & $\begin{array}{l}\text { - Implementation of clinical studies } \\
\text { - Detection of cases outside endemic countries } \\
\text { - Increase awareness / case reporting among clinicians }\end{array}$ \\
\hline Pharmaceutical industry & $\begin{array}{l}\text { - Data on drug sales with sufficient detail allowing geographically high resolution in } \\
\text { leishmaniasis endemic areas } \\
\text { - Funding }\end{array}$ \\
\hline
\end{tabular}

varies by type of TNF alpha antagonist. As pointed out before, this was possible by combining published information from different European countries. Based hereon, it becomes obvious that fragmentation of data at national level hampers a better description of the safety profile of TNF alpha antagonists with regard to rare opportunistic infections. Therefore, evaluation of data across registers and at European level has been suggested to increase the ability to detect such events [13]. Although this approach has potential to contribute to our understanding of opportunistic leishmaniasis, a range of challenges remain.

Firstly, transmission of leishmaniasis occurs geographically in confined foci since it is bound to the presence of the phlebotomine vector. This is reflected in the large variation of sero-prevalence reported in regions around the Mediterranean basin $[2,3,5]$. Hence, to further advance our knowledge on opportunistic leishmaniasis in patients receiving biotherapies, it will be crucial that national drug registers adequately cover the population in areas where transmission occurs. The variation in transmission risk also implies that estimates of the absolute risk of leishmaniasis in TNF alpha antagonist users calculated from register data will vary according to the proportion of subjects live in endemic regions enrolled in the register.

Secondly, European national drug registers on the safety of TNF alpha antagonist drugs and other biotherapies use different methodology and do not record data in a uniform manner. To facilitate collaboration between national drug registers and to allow supranational data analyses, harmonisation of European registers is a prerequisite. A uniform reporting scheme has so far only been adopted by Great Britain, Sweden, and Germany, but not by France and Spain, countries where leishmaniasis is endemic [13].

Thirdly, it is unclear, whether all countries with leishmaniasis endemic regions have an active national drug register or cohort. For instance, from a number of countries in the Balkan region, there are no peer-reviewed publications available on PubMed reporting on such activities.

The Table outlines potential components of a coordinated research effort on opportunistic leishmaniasis in Europe and Box 2 the resulting strengths.

\section{Conclusions}

Although the initiation of national registers with support of the pharmaceutical industry was a major step forward in better defining the safety profile of TNF alpha antagonist drugs and other biotherapies, there are shortcomings of this system with regard to opportunistic leishmaniasis. Crucial for improved case detection and reduced underreporting will be a closer link between existing national drug registers and institutions specialised in the diagnosis and therapy of leishmaniasis, e.g. tropical medicine institutes, dermatology departments, etc. This will likely enable the detection of a considerable number of additional cases of opportunistic leishmaniasis that are either directly seen at these institutions or were suspected elsewhere but laboratory confirmed there. Using existing network structures in Europe such as TropNet (www.tropnet.net) and EuroTravNet (www.istm.org/eurotravnet/ 


\section{Box 2}

Strengths of a coordinated research effort at European level on opportunistic leishmaniasis in patients treated with tumor necrosis factor alpha antagonists

- Impact - a coordinated research effort will allow estimating the public health impact of tumor necrosis factor alpha (TNF) alpha antagonists on leishmaniasis in Europe.

- Statistical power - a coordinated research effort will increase statistical power through improved case detection and more comprehensive coverage of populations in endemic regions.

- Defragmentation - a coordinated research effort will ensure that data available is not restricted to single countries.

- Standardisation - a coordinated research effort will improve database quality for supra-national analysis through uniform methodology, reporting scheme, and common denominators (e.g. data on drug sales).

- Flexibility - a coordinated research effort will allow rapid incorporation of newly emerging biotherapies and rare infections other than leishmaniasis.

main.html) could facilitate the creation of such links. An extended network of clinical specialists in rheumatology, infectious diseases, tropical medicine, and dermatology will be necessary to answer the research questions pertaining to the clinical management of patients with opportunistic leishmaniasis set out in this paper.

Prospective data from a well-defined population at risk of acquiring or being latently infected with leishmania will be needed to obtain meaningful estimates of the incidence of clinical manifest infection that can be attributed to TNF alpha antagonist use and to evaluate the clinical role of screening for latent infection. This can only be achieved through focused post-marketing safety studies that make use of large cohorts in areas endemic for leishmaniasis. To ensure uniform methodology and data collection, such activity should be designed and coordinated at European level before being implemented by the existing national registers. In European countries endemic for leishmaniasis but without a well-functioning register or cohort, this may require enhancing or newly creating such structures. Taken together, these activities will need adequate funding and most importantly, substantial collaboration across disciplines including input from epidemiologists and public health experts.
References

1. Scallon B, Cai A, Solowski N, Rosenberg A, Song XY, Shealy $D$, et al. Binding and functional comparisons of two types of tumor necrosis factor antagonists. J Pharmacol Exp Ther. 2002;301(2):418-26.

http://dx.doi.org/10.1124/ipet.301.2.418

2. Diza E, Kansouzidou A, Gerou S, Vezyri E, Metallidis S, Antoniadis A. Leishmaniases in northern greece: Seroprevalence of the infection and incidence of the disease during the period 2001-2006. Eur J Clin Microbiol Infect Dis. 2008;27(10):997-1003.

http://dx.doi.org/10.1007/s10096-008-0538-y PMid: 18512088

3. Federico G, Damiano F, Caldarola G, Fantini C, Fiocchi V, Ortona L. A seroepidemiological survey on leishmania infantum infection. Eur J Epidemiol. 1991;7(4):380-3. http://dx.doi.org/10.1007/BF00145003

4. Ready PD. Leishmaniasis emergence in europe. Euro Surveill. 2010;15(10):19505. (10):pii=19505. Available from: http://www. eurosurveillance.org/ViewArticle.aspx?Articleld=19505

5. Moral L, Rubio EM, Moya M. A leishmanin skin test survey in the human population of l'alacanti region (spain): Implications for the epidemiology of leishmania infantum infection in southern europe. Trans R Soc Trop Med Hyg. 2002;96(2):12932.

http://dx.doi.org/10.1016/S0035-9203(02)90278-6

6. Zanger P, Kötter I, Kremsner PG, Gabrysch S. Tumor necrosis factor alpha antagonist drugs and leishmaniasis in europe. Clin Microbiol Infect. 2012;18(7):670-6. http://dx.doi.org/10.1111/j.1469-0691.2011.03674.x PMid:21985586

7. Xynos ID, Tektonidou MG, Pikazis D, Sipsas NV. Leishmaniasis, autoimmune rheumatic disease, and anti-tumor necrosis factor therapy, europe. Emerg Infect Dis. 2009;15(6):956-9. http://dx.doi.org/10.3201/eid1506.090101 PMid:19523302 PMCid:PMC2727307

8. Wallis RS, Broder MS, Wong JY, Hanson ME, Beenhouwer DO. Granulomatous infectious diseases associated with tumor necrosis factor antagonists. Clin Infect Dis. 2004:38(9):1261-5. http://dx.doi.org/10.1086/383317 PMid:15127338

9. Tubach F, Salmon D, Ravaud P, Allanore Y, Goupille P, Bréban $M$, et al. Risk of tuberculosis is higher with anti-tumor necrosis factor monoclonal antibody therapy than with soluble tumor necrosis factor receptor therapy: The three-year prospective french research axed on tolerance of biotherapies registry. Arthritis Rheum. 2009;60(7):1884-94. http://dx.doi.org/10.1002/art.24632 PMid:19565495 PMCid:PMC2921546

10. Allenbach C, Launois P, Mueller C, Tacchini-Cottier F. An essential role for transmembrane tnf in the resolution of the inflammatory lesion induced by leishmania major infection. Eur J Immunol. 2008;38(3):720-31.

http://dx.doi.org/10.1002/eji.200737662 PMid:18266271

11. Lohmann-Matthes ML, Luttig B, Hockertz S. Involvement of membrane-associated tnf in the killing of leishmania donovani parasites by macrophages. Behring Inst Mitt. 1991;88:125-32. PMid:2049029

12. Zanger P, Kötter I, Raible A, Gelanew T, Schonian G, Kremsner PG. Successful treatment of cutaneous leishmaniasis caused by leishmania aethiopica with liposomal amphothericin $\mathrm{b}$ in an immunocompromised traveler returning from eritrea. Am J Trop Med Hyg. 2011;84(5):692-4.

http://dx.doi.org/10.4269/ajtmh.2011.10-0712 PMid:21540377 PMCid:PMC3083735

13. Zink A, Askling J, Dixon WG, Klareskog L, Silman AJ, Symmons DP. European biologicals registers: Methodology, selected results and perspectives. Ann Rheum Dis. 2009;68(8):1240-6. http://dx.doi.org/10.1136/ard.2008.091926 PMid:18647854 